\title{
Techniques to Improve Power Amplifier Energy Efficiency for $5 \mathrm{G}$
}

\author{
Erdem Bala, Leonid Kazakevich, Rui Yang \\ InterDigital Communications, Inc., Melville, NY
}

\begin{abstract}
Improved spectral and energy efficiency are two key requirements for the fifth generation (5G) wireless communication systems. The inefficiency and non-linearity of traditional power amplifiers (PA) pose significant challenges to meet these requirements, especially for multicarrier modulation signals (MCM) with high peak to average power ratio (PAPR). In this paper, we study methods to improve the energy efficiency of PAs and compensate for the distortion of PA non-linearity. A low complexity envelope tracking (ET) method where the supply voltage of the PA consists of two or more discrete levels is presented. To reduce the distortion due to PA nonlinearity, a power amplifier model with power supply variation is introduced and a digital predistortion technique based on a Volterra series is used. Finally, an integrated architecture combining crest factor reduction (CFR), DPD, and ET is presented. Simulations and measurements from a hardware platform demonstrate the energy efficiency gains attained by the proposed techniques.
\end{abstract}

Index Terms-OFDM, multicarrier modulation, power amplifier, energy efficiency, spectral efficiency, envelope detection, digital predistortion

\section{INTRODUCTION}

5G wireless communication systems will be designed to provide huge capacity, which will require significant increases in spectral efficiency and available bandwidth. In addition to capacity, another key requirement is energy efficiency. Several candidate technologies such as mmWave communication, massive MIMO, ultra-dense small cells among others have been proposed to meet the requirements of these next generation networks [1].

Orthogonal Frequency Division Multiplexing (OFDM) is currently the most widely used multi carrier modulation technique; it has been adopted in many wireless communication standards including 802.11 [2] and 3GPP Long Term Evolution (LTE) [3]. Several alternative multicarrier modulation waveforms have been proposed for 5G [4]-[8] and one common goal of these waveforms is to reduce the out-ofband emission to make spectrum sharing more efficient.

A common drawback of MCM signals is high peak to average power ratio (PAPR). When a high PAPR signal passes through a nonlinear power amplifier (PA), intermodulation distortion is generated, creating out-of-band emission that can interfere with other transmission in adjacent bands. In addition, in-band distortion, which degrades the bit error rate (BER) performance, occurs. Waveforms with high PAPR present a challenge to the design of power amplifiers in battery operated devices such as mobile wireless devices where power consumption is critical. High linearity is required to prevent distortion: to reduce the impact from PA nonlinearity, a common technique is to back off the input signal in order to have it fit within the linear dynamic range of the PA. However, this method will lead to very low PA efficiency, typically less than $10 \%$ for MCM signals.

To avoid heavily backing off the signal, two methods may be used: reducing the PAPR of the baseband signal and PA linearization [9]. There have been many PAPR reduction methods developed in the literature with varying tradeoffs [10], [11]. In this paper, we consider both methods, but focus more on PA linearization techniques with a simple PAPR reduction algorithm in the baseband (i.e., clipping and filtering, also known as crest factor reduction (CFR)). Among all linearization techniques, digital predistortion (DPD) is one of the most cost effective options. A digital pre-distorter in the baseband creates an expanding nonlinearity that is complementary to the compressing characteristic of the power amplifier. Digital predistortion (DPD) has attracted much attention for OFDM [9] and has also been used to compensate amplitude and phase nonlinear distortions of OFDM-OQAM signals in [12], where a memoryless PA model has been used.

Another significant issue in dealing with high PAPR signals is PA efficiency [13]. Fig. 1 from [14] plots the instantaneous efficiency against output power across a range of supply voltages for a conventional handset PA. With constant supply voltage, the power amplifier efficiency is highest at maximum output power and is reduced as the average output power is reduced. Several techniques such as envelope elimination and restoration [15], envelope tracking (ET) [15], the Doherty amplifier technique [16], and PA switching [17] have been proposed to enhance the PA efficiency. Among these, envelope tracking is the most suitable approach for wireless devices due to simplicity; it uses only one PA and minimal additional circuitry. In this technique, the supply voltage is varied as a function of instantaneous output power to ensure that the device is always operating at the maximum possible efficiency. As shown in Fig. 1, the envelope tracking efficiency locus always delivers better performance than the fixed supply efficiency locus while maintaining high linearity [14].

Two types of ET methods proposed in the literature are continuous envelope tracking (CET) [18] and reduced 
bandwidth continuous envelope tracking (RB CET) [19]. Continuous tracking requires a high speed power supply, which is done by paralleling outputs of a DC/DC converter and a high speed linear amplifier. This type of ET has high cost and the power supply efficiency may suffer due to power dissipation in the linear amplifier. In reduced bandwidth tracking, the filtered signal envelope is used to modulate the power supply of the PA with lower than continuous tracking speed. Although it has lower cost than continuous tracking, the cost is still too high for most mobile devices. One method to reduce the ET complexity is to incorporate two or more discrete input voltage levels [20].

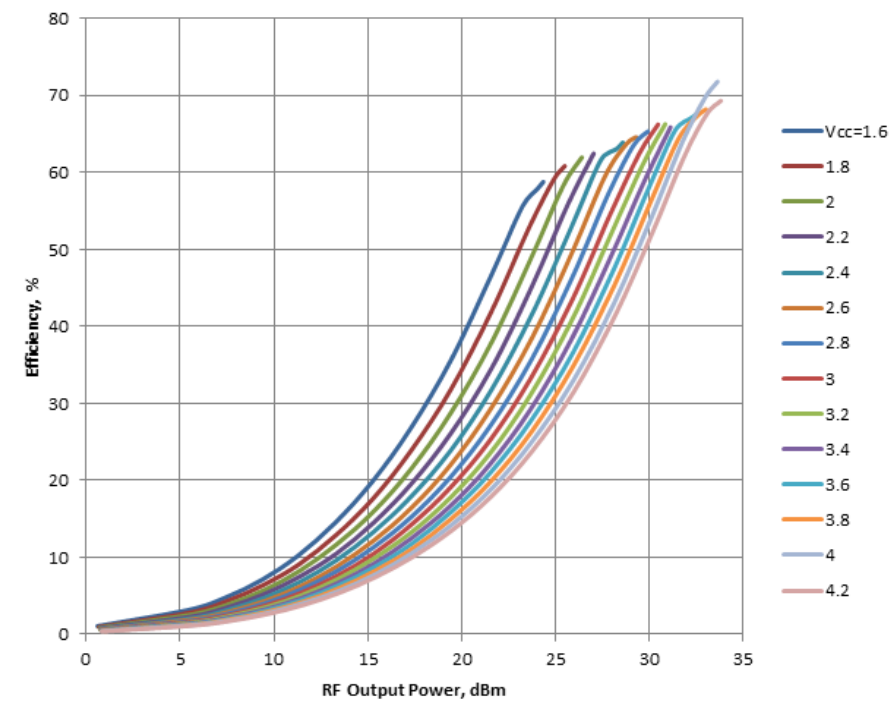

Figure 1 Efficiency for a range of supply voltages

In this paper, we study methods to improve PA efficiency and reduce the distortion due to PA non-linearity. A discrete ET method that is integrated with DPD is presented. With this technique, a wider range of discrete voltage levels than [20] can be supported and the battery is used for the high level voltage without the need of a DC/DC converter. The presented DPD technique uses a Volterra series based PA model that incorporates power supply variation. The model is characterized with measurements from a commonly used UE PA (RF3266 [21]). In addition to the above, an integrated solution that includes CFR, DPD, and ET is presented and evaluated with measurements taken from a hardware platform that was built to test the proposed algorithms.

\section{DYNAMIC SUPPLY VOLTAGE PA}

\section{A. Discrete Envelope Detection}

Tracking the transmitted signal envelope and providing minimum usable DC voltage to the PA improves the overall PA power efficiency. Fig. 2 illustrates the timing diagrams for a conventional fixed supply voltage (no ET), CET, RB CET, and the discrete ET scheme with 3 input voltage levels. The area that lies between the envelope and the supply voltage line determines the energy dissipated as heat.

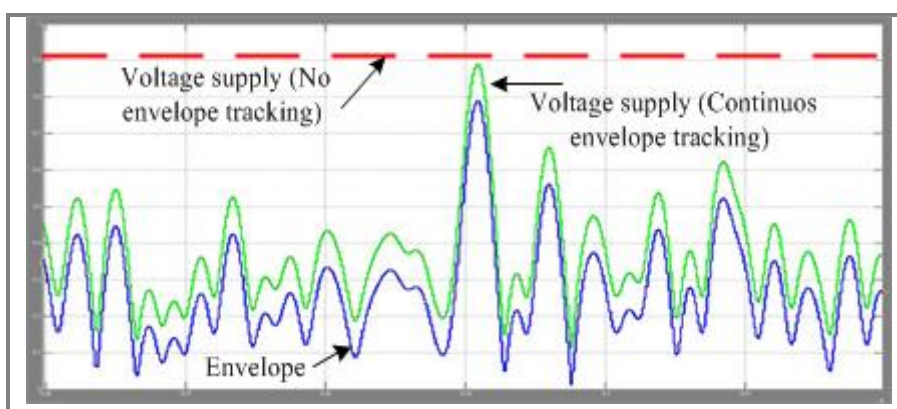

(a) Timing diagram without envelope tracking and with continuous envelope tracking

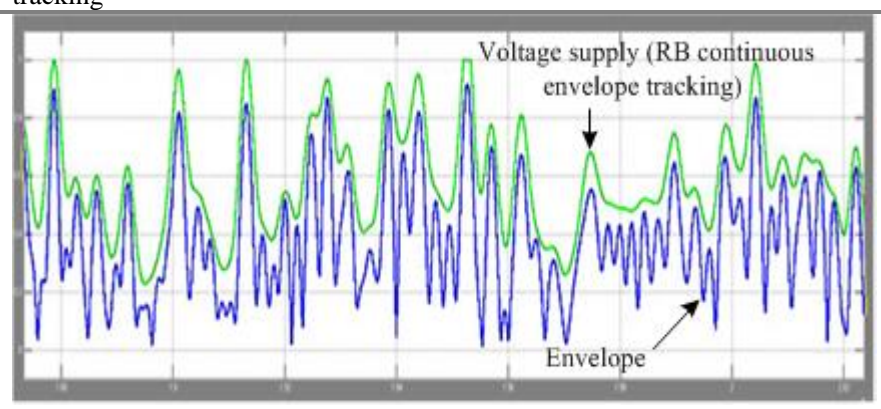

(b) Timing diagram of reduced bandwidth continuous envelope tracking

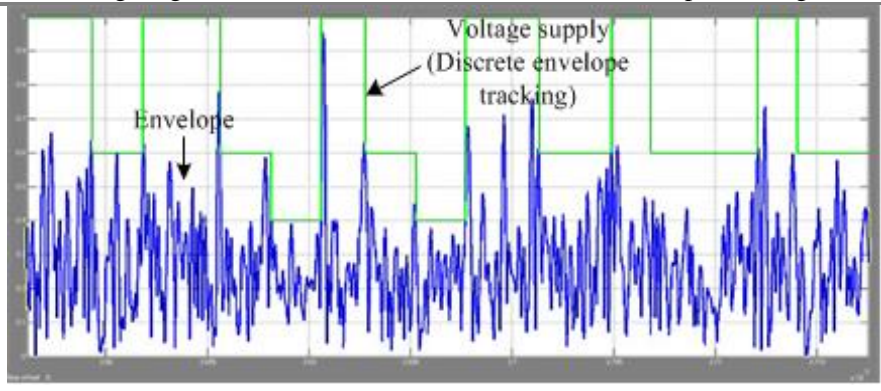

(c) Timing diagram of 3-level discrete envelope tracking

Figure 2 Timing diagrams of various envelope tracking methods(x axis: time; y-axis: amplitude)

Continuously variable power supply voltage control offers the greatest PA efficiency but the complexity of the control algorithm makes it less appealing than a simpler discrete power supply voltage control. When using discrete envelope detection, the optimal values of the discrete Vcc values are chosen to maximize the PA efficiency and may be adjusted according to the adaptation of the RF output power. These values should not be less than the minimal value $\mathrm{V}_{\text {ccmin }}$, which is slightly above $1 \mathrm{~V}$ for a conventional handset PA.

The performance of the proposed step ET technique is illustrated in Fig. 3 and compared with the alternative techniques assuming an ideal DC/DC converter and a DC/DC converter with $70 \%$ efficiency. We can see that the efficiency of 2-level discrete ET with CFR is better than reduced bandwidth ET with a 70\% efficient DC/DC converter. In the mid RF output power range, the 2-level discrete ET performs as well as the continuous ET and reduced bandwidth ET with ideal DC/DC converters. 


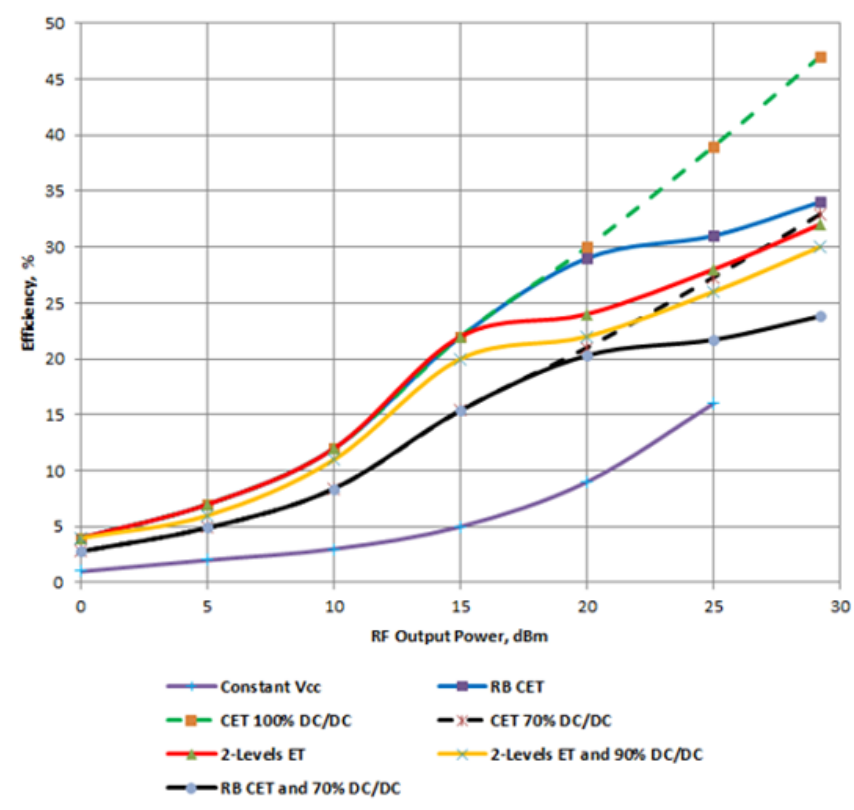

Figure 3 Efficiency of PA with discrete and reduced bandwidth ET

\section{Digital PRE-Distortion (DPD) TeChNiQues}

\section{A. Suggested PA Model Incorporating Power Supply Variation}

In this section, we propose a PA model that contains a static nonlinearity block and a feedback loop with an IIR filter to simulate memory effects. A top level block diagram of the model is shown in Fig. 4. The static nonlinearity property is implemented using a memoryless polynomial with selectable order. For simulation purposes, a $7^{\text {th }}$ order has been used with a coefficient set taken from [22] which correspond to performance of a solid-state PA with moderate distortion. The polynomial order can be decreased and the coefficient values can be changed in order to introduce heavier distortions and/or simplify DPD implementation. The lowpass filter used to introduce memory effects is a simple first or second order filter.

To model the effect of power supply variation on PA nonlinearity, we define $v=\frac{V}{V_{\max }}$ as a scaling coefficient where the PA supply voltage level is $V$ and the maximum operating voltage is $V_{\max }$. The amplitude distortion components of the polynomial PA model is predicted to rise inversely proportional to the square of $v$, while the phase distortion component produced by the polynomial model is expected to be inversely proportional to the square root of $v$. The final equation defining the static nonlinearity block output takes the following form:

$y_{n l}[k]$
$=\left.\left|\frac{1}{v^{2}} \sum_{n=0}^{N} \alpha_{n}\right| x[k]\right|^{n} \mid e^{\left\{<(x[k])+\frac{1}{\sqrt{v}}<\left(\frac{1}{v^{2}} \sum_{n=0}^{N} \alpha_{n}|x[k]|^{n}\right)\right\}}$ where $x[k]$ is the input digital sample, $\alpha_{n}$ is the $\mathrm{n}$-th polynomial coefficient, $\angle(x)$ represents the phase of a complex number $x$ in radius.

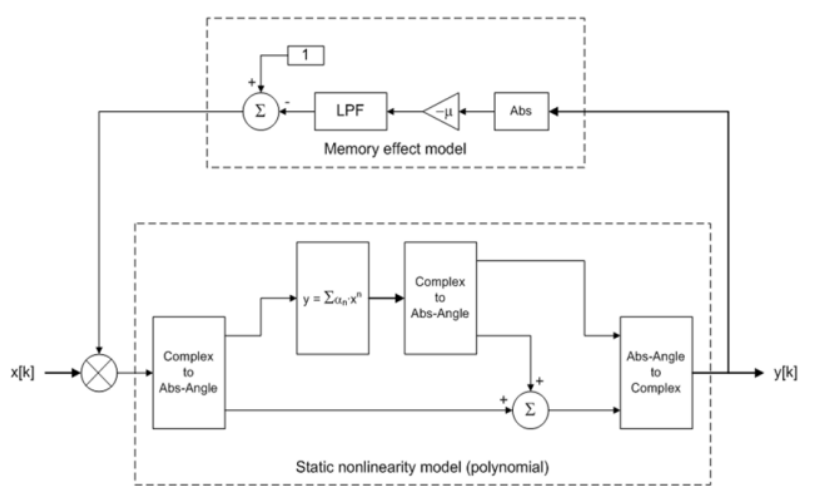

Figure 4 Block diagram of the proposed PA model

In order to incorporate measurements to the model, a set of $\mathrm{AM}-\mathrm{AM}$ and AM-PM distortion curves are measured for a number of supply voltage levels with input signal magnitude swept over the whole PA operating range. For each pair of AM-AM and AM-PM curves, complex coefficients of the polynomial PA model are extracted by means of least square fitting. Once a set of polynomial coefficients is obtained for each supply voltage level, polynomial approximations of individual coefficients can be found while using the voltage supply as a variable.

The resultant PA model is verified by comparing it to measurements taken from the RF3266 PA module which operates in the $1.9 \mathrm{GHz}$ band. Fig. 5 and Fig. 6 show the measured and approximated AM-AM and AM-PM responses, respectively. As can be seen from the figures, quite good correspondence between real and approximated distortion curves can be achieved over most of the input power and supply voltage range. The approximation results in large errors in the regions where the input signal power exceeds the normal operating range for a given supply voltage level. This, however, doesn't undermine validity of the model because such situations are not possible in practice.

\section{B. Digital Predistortion}

The goal of digital pre-distortion is to transform the desired digital signal $x[k]$ in such a way that the scaled baseband equivalent of the PA output, $y[k]$, would be exactly equal to $x[k]$. Ideally, the nonlinearity introduced by a digital predistorter, $F_{D P D}(x)$, should be the inverse of the nonlinearity introduced by a PA, $F_{P A}(x)$. In this work, a Volterra series based pre-distorter is chosen since it compensates both static nonlinearity and memory effects of the PA [23], [24]. Since increasing the number of time domain samples and/or the polynomial order causes the number of coefficients in the Volterra series to grow dramatically, in practice, a simplified version of the Volterra series is used. One of the common choices for a truncated Volterra series is a memory polynomial model [25]: 


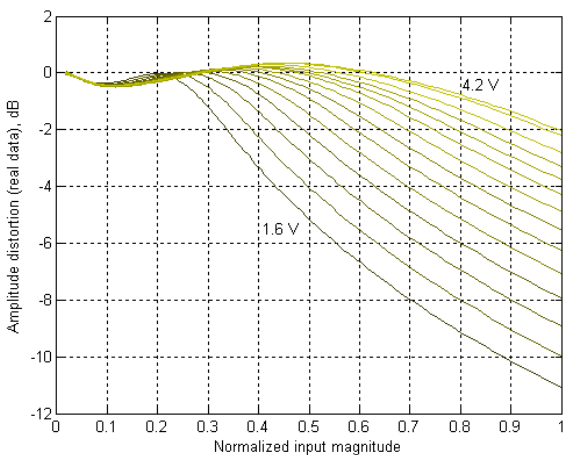

Figure 5-1 AM-AM response (measured data)

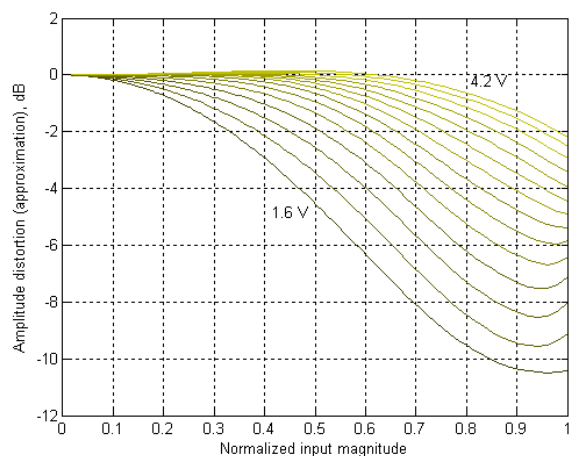

Figure 5-2 AM-AM response (approximation)

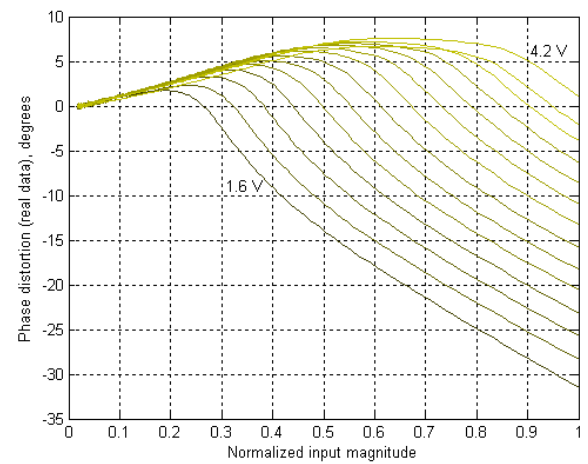

Figure 6-1 AM-PM response (measured data)

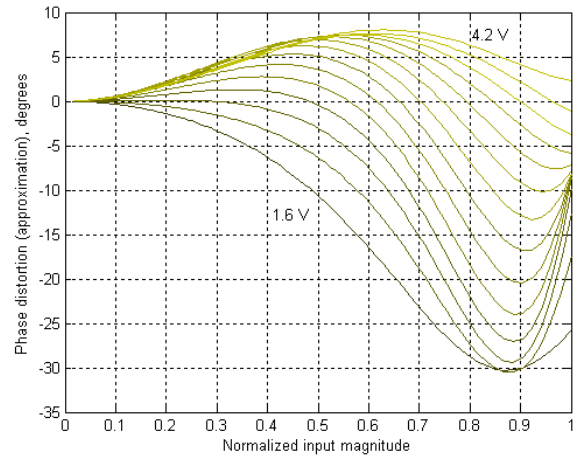

Figure 6-2 AM-PM response (approximation)

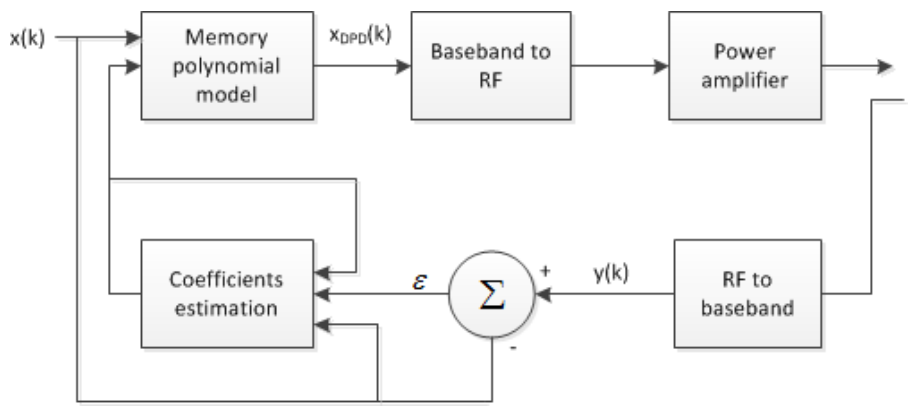

Figure 7 Truncated Volterra series based MRAS pre-distortion architecture

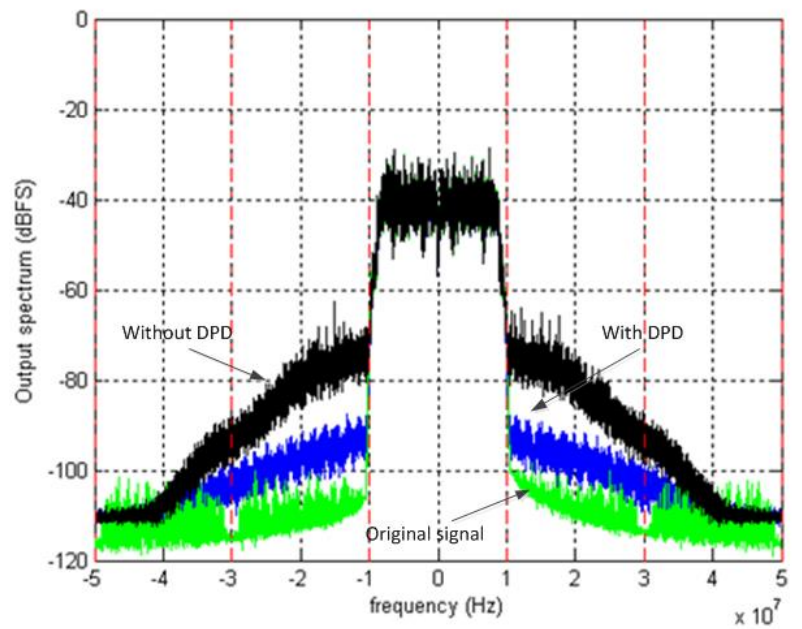

Figure 8 Predistortion performance (output spectrum): green: original signal, black: baseband equivalent of PA output without DPD, blue: baseband equivalent of PA output with DPD

$$
x_{D P D}[k]=\sum_{p=1}^{P} \sum_{m=0}^{M} a_{p, m} \cdot|x[k-m]|^{p-1} \cdot x[k-m]
$$

where $P$ is the polynomial order, $M$ is the memory depth and $a_{p, m}$ are the model coefficients. The goal of a pre-distorter based on a memory polynomial model is to estimate coefficients $a_{p, m}$ and adaptively update them in order to account for memory effects. A block diagram of a memory polynomial Model Reference Adaptive System (MRAS) [26] pre-distorter is shown in Fig. 7.

The MRAS pre-distorter adaptively tunes the Volterra series coefficients in order to minimize $\varepsilon$, the error signal shown in Fig. 7. This optimization can be achieved be minimizing the following cost function:

$$
\sum_{k}\left|\varepsilon[k]-\sum_{i} \Delta a_{i} \gamma_{i}\{x[k]\}\right|^{2}
$$

where $k$ is the sample index, $i$ is the index that includes both $m$ and $p$ indexes, $\Delta a_{i}$ are the current coefficient errors which are to be computed, and $\gamma_{p, m}(x)=|x[k-m]|^{p-1} x[k-m]$. For each data buffer, a new set of coefficient errors is calculated using an LMS algorithm and the DPD coefficients are updated recursively using the formula $a_{i}[(n+1) T]=a_{i}[n T]-\mu \Delta a_{i}$, 
where $T$ is the time required for data accumulation and coefficient estimation.

Fig. 8 illustrates the output spectrum of the PA with and without DPD when the input signal is OFDM-OQAM. The green line corresponds to the input signal. The black line is the spectrum of the PA output without pre-distortion (a crest factor reduction technique is applied and the CFR block output is amplified by the CFR reduction amount to align peaks). The blue line shows the spectrum of the PA output with DPD. The buffer length of 64 samples is used in the simulation and each $64^{\text {th }}$ buffer is processed by the coefficient estimator. We can see from the figure that the out-of-band emission of the input signal increases significantly due to the nonlinearity of the PA. When DPD is applied, considerable reduction in the spectral leakage is achieved.

\section{INTEGRATED ARCHITECTURE}

An integrated architecture encompassing CFR, DPD, and ET has been designed and built to evaluate the proposed techniques. The high level block diagram of the architecture is illustrated in Fig. 9 and a photograph of the hardware platform is shown in Fig. 10. The platform can generate signals based on 802.11ac [27] or the LTE uplink [3].

Fig. 11 shows the measurements taken from the platform for a $20 \mathrm{MHz} 802.11$ ac signal while Fig. 12 shows the measurements for a $10 \mathrm{MHz}$ LTE uplink signal. The figures illustrate PA efficiency and error vector magnitude (EVM) which is a measure of the effect of nonlinearity on the accuracy of detection at the receiver. It is defined as the magnitude of the difference between the desired and the actual received signal vectors and is usually expressed as a fraction or percentage of the signal amplitude. We can see from these figures that the proposed combination of CFR, DPD and Step ET provides more than 2 times gain in PA efficiency compared to the reference PA. In addition, 6-8 dB of gain in maximum RF output power can be attained at 5\% EVM.

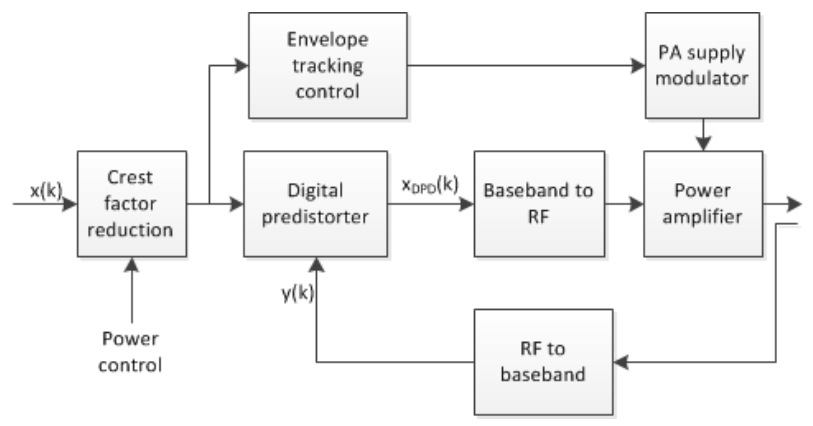

Figure 9 Block diagram of the proposed architecture

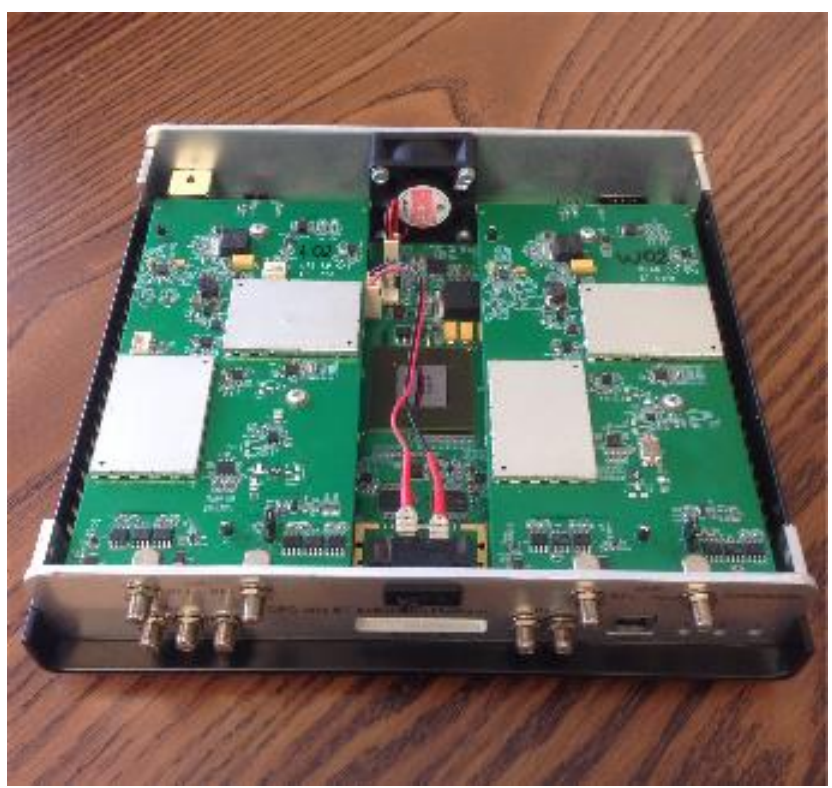

Figure 10 The hardware platform built to evaluate the proposed techniques

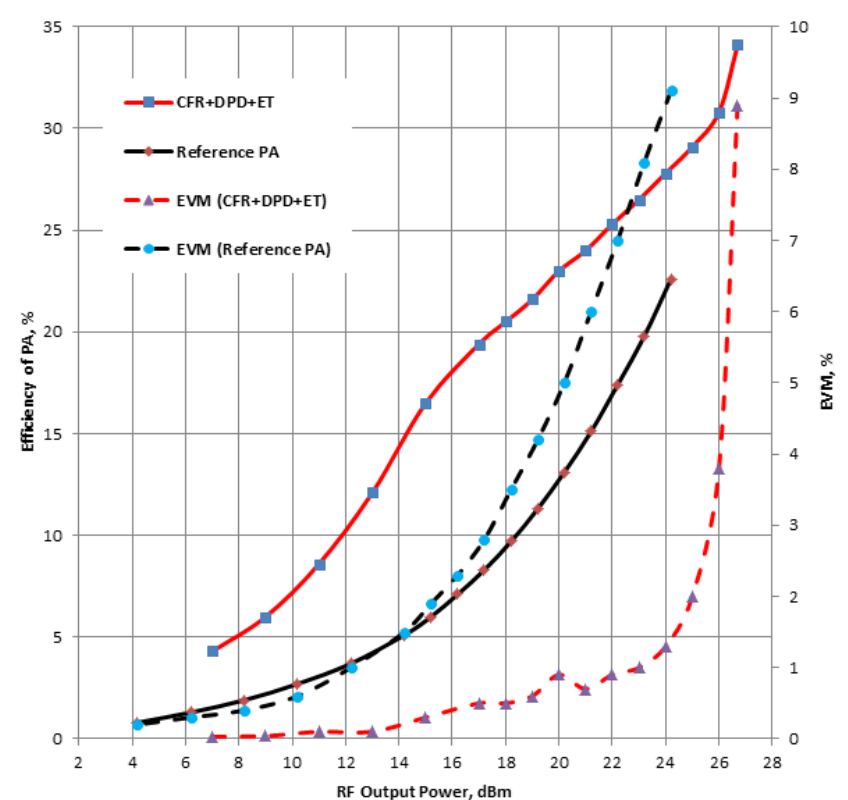

Figure 11 PA efficiency measurements from the hardware platform for 802.11ac signal 


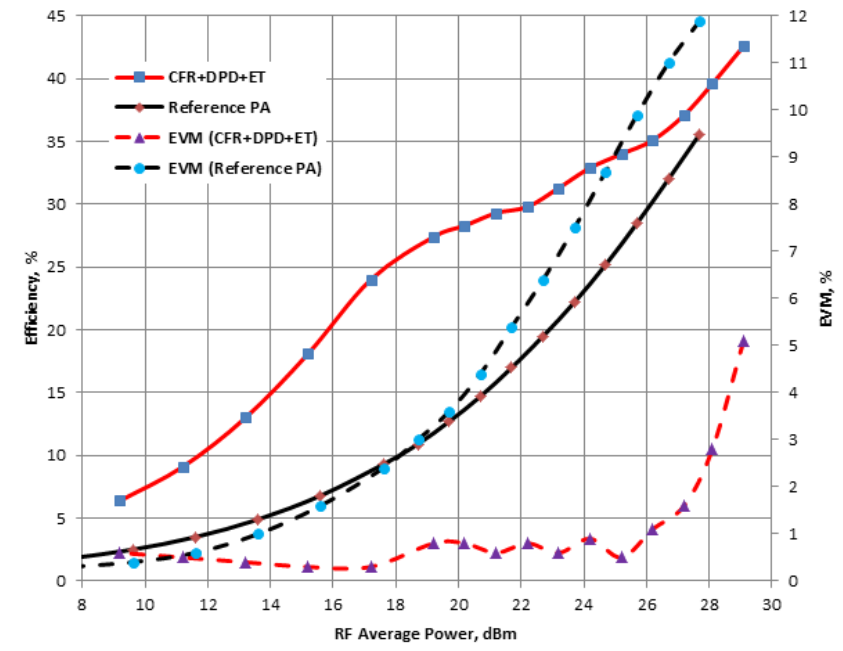

Figure 12 PA efficiency measurements from the hardware platform for LTE signal

\section{CONCLUSIONS}

In this paper, we have studied methods to improve PA energy efficiency and compensate for the distortion introduced by the PA non-linearity. A low complexity envelope tracking method that uses a discrete supply voltage and a digital predistortion technique has been presented. The proposed methods were developed by using a PA model encompassing power supply variation and memory effects. In addition, we have presented an integrated architecture combining crest factor reduction, digital predistortion, and envelope tracking. These methods were implemented on a hardware platform. Simulations and measurements have shown that the proposed techniques can significantly improve the PA efficiency for MCM signals.

\section{REFERENCES}

[1] J.G. Andrews, S. Buzzi, W. Choi, S.V. Hanly, A. Lozano, A. C. K. Soong, J.C. Zhang, "What Will 5G Be?," Selected Areas in Communications, IEEE Journal on , vol.32, no.6, pp.1065,1082, June 2014.

[2] "Specific requirements- Part 11: Wireless LAN Medium Access Control (MAC) and Physical Layer (PHY) Specifications," IEEE Standard 802.11, 2012.

[3] E-UTRA; Physical channels and modulation, 3GPP TS36.211 V8.9.0., 2009-12.

[4] G. Fettweis, M. Krondorf, and S. Bittner, "GFDM-Generalized frequency division multiplexing," in Proc. IEEE Veh. Technol. Conf., Apr. 2009, pp. 1-4.

[5] V. Vakilian, T. Wild, F. Schaich, S. ten Brink, and J.-F. Frigon, "Universal-filtered multi-carrier technique for wireless systems beyond LTE," in Proc. IEEE GLOBECOM Broadband Wireless Access Workshop, Dec. 2013, pp. 223-228.

[6] J. Li, E. Bala, R Yang, "Resource block Filtered-OFDM for future spectrally agile and power efficient systems," Physical Communication 11: 36-55 (2014)

[7] P. Siohan, C. Siclet and N. Lacaille, "Analysis and design of OFDM/OQAM systems based on filterbank theory," Signal Processing, IEEE Transactions on, vol.50, no.5, pp.1170-1183, May 2002.

[8] B. Farhang-Boroujeny, "OFDM versus filter bank multicarrier," IEEE Signal Processing Magazine, pp. 92-112, May 2011.
[9] L. Ding, "Digital Predistortion of Power Amplifiers for Wireless Applications," Ph.D. dissertation, Dept. Elect. And Comp. Eng., Georgia Institute of Technology., GA, 2004

[10] X. Li and L. J. Cimini Jr., "Effects of clipping and filtering on the performance of OFDM," IEEE Communications Letters, Vol. 2, pp. 131-133, May 1998.

[11] S.H. Muller and J.B.Huber, "A Comparison of Peak Power Reduction Schemes for OFDM," Proceedings of IEEE GLOBECOM, Volume 1, 1997.

[12] R. Zayani, Y. Medjahdi, H. Bouhadda, H. Shaiek, D. Roviras and R. Bouallegue, "Adaptive Predistortion techniques for non-linearly amplified FBMC-OQAM signals", IEEE VTC'Spring, Seoul, May 2014.

[13] J. Joung, C. K. Ho, S. Sun, "Spectral Efficiency and Energy Efficiency of OFDM Systems: Impact of Power Amplifiers and Countermeasures," Selected Areas in Communications, IEEE Journal on , vol.32, no.2, pp.208,220, February 2014.

[14] S. Baker, "An introduction to envelope tracking for RF amplifiers," OpenET Alliance White Paper, 2011.

[15] P.A. Dal Fabbro, M. Kayal, Linear CMOS RF Power Amplifiers for Wireless Applications. Springer, 2010.

[16] S. C. Cripps, RF Power Amplifiers for Wireless Communications. Artech House, 1999, p 225-235.

[17] J. Joung, C. K. Ho, S. Sun, "Power Amplifier Switching (PAS) for Energy Efficient Systems," Wireless Communications Letters, IEEE , vol.2, no.1, pp.14,17, February 2013.

[18] G. Wimpenny, "Improving Multi-carrier PA Efficiency Using Envelope Tracking", Microwave Journal, November, 2008.

[19] J. Jeong, D.F. Kimball, M. Kwak, C. Hsia, P. Draxler, P.M. Asbeck, "Wideband envelope tracking power amplifier with reduced bandwidth power supply waveform," Microwave Symposium Digest, pp.1381,1384, June 2009.

[20] A. Khanifar, N. Maslennikov, M. Gurvich, B. Vassilakis, "Systems and Methods of Dynamic Bias Switching for Radio Frequency Power Amplifiers," US Patent \#6,937,094, Aug. 30, 2005.

[21] http://ir.rfmd.com/releasedetail.cfm?ReleaseID=393706

[22] S.C. Cripps. Advanced Techniques in RF Power Amplifier Design. Artech House, 2002.

[23] J. S. Kenney, W. Woo, L. Ding, R. Raich, H. Ku and G. T. Zhou, "The impact of memory effects on predistortion linearization of RF power amplifiers," in Proc. Int. Symp. Microwave Optical Technol., pp. 189193, June 2001.

[24] S. Benedetto, E. Biglieri and R. Daffara, "Modeling and performance evaluation of nonlinear satellite links - a Volterra series approach," IEEE Trans. Aerosp. Electron. Syst., vol. AES-15, pp. 494-507, July 1979.

[25] J. Kim and K. Konstantinou, "Digital predistortion of wideband signals based on power amplifier model with memory," Electron. Lett., vol. 37, pp. 1417-1418, Nov. 2001.

[26] K.S. Narendra and A.M. Annaswamy, Stable Adaptive Systems. Dover Publications, 2005.

[27] "Specific Requirements-Part 11: Wireless LAN Medium Access Control and Physical Layer Specifications - Amendment 4: Enhancements for Very High Throughput for operation in bands below $6 \mathrm{GHz}$," IEEE Standard 802.11ac, 2013. 